\title{
Rainfastness of agrochemical formulations based on $N$-vinyl pyrrolidone polymers and their interpolymer complexes with poly(acrylic acid)
}

Article

Accepted Version

Creative Commons: Attribution-Noncommercial-No Derivative Works 4.0

Sevastos, A. A., Thomson, N. R., Lindsay, C., Padia, F. and Khutoryanskiy, V. V. (2020) Rainfastness of agrochemical formulations based on $\mathrm{N}$-vinyl pyrrolidone polymers and their interpolymer complexes with poly(acrylic acid). European Polymer Journal, 134. 109852. ISSN 0014-3057 doi: https://doi.org/10.1016/j.eurpolymj.2020.109852 Available at https://centaur.reading.ac.uk/91349/

It is advisable to refer to the publisher's version if you intend to cite from the work. See Guidance on citing.

To link to this article DOI: http://dx.doi.org/10.1016/j.eurpolymj.2020.109852

Publisher: Elsevier

All outputs in CentAUR are protected by Intellectual Property Rights law, including copyright law. Copyright and IPR is retained by the creators or other copyright holders. Terms and conditions for use of this material are defined in the End User Agreement. 


\section{www.reading.ac.uk/centaur}

\section{CentAUR}

Central Archive at the University of Reading

Reading's research outputs online 


\title{
Rainfastness of agrochemical formulations based on
}

\section{$\mathrm{N}$-vinyl pyrrolidone polymers and their interpolymer}

\author{
complexes with poly(acrylic acid)
}

Apostolos A. Sevastos $\nmid, \neq$, Niall R. Thomson $\neq$, Christopher Lindsay $\neq$, Faheem Padia $\neq$,

Vitaliy V. Khutoryanskiył*

$\dagger$ Reading School of Pharmacy, University of Reading, Whiteknights, P.O. Box 224, Reading RG6 6AD, U.K.

†Syngenta, Jealott’s Hill International Research Centre, Bracknell, Berkshire RG42 6EY, U.K.

* Prof Vitaliy V. Khutoryanskiy.

Corresponding Author at Reading School of Pharmacy, The University of Reading,

Whiteknights, P.O. Box 224, Reading RG6 6AD, U.K., email:

v.khutoryanskiy@reading.ac.uk

\section{ABSTRACT}

In this study, poly(N-vinyl pyrrolidone) (PVP), a cheap, safe and non-toxic polymer, was explored using a range of analytical methods including fluorescence microscopy to gain insight into the role of polymer physicochemical properties on rainfastness, i.e. tenacity of foliar deposits against rain, 
of agrochemicals on plant surfaces. Three methods were approached to increase rainfastness of PVP, i.e. using high molecular weight grades of the polymer, pre- blending PVP with poly(acrylic acid) (PAA) and successively depositing drops of each polymer (PVP or PAA) on top of the other. Regarding the first method, from the different commercial grades of PVP studied, it was revealed that the polymer with highest molecular weight $(1300 \mathrm{kDa})$ significantly improved the rainfastness of a model fungicide (azoxystrobin). The rainfastness results correlated with film dissolution in water. In the second method, rainfastness properties of PVP were improved by mixing it with PAA and it was shown that PVP-PAA mixtures at the 50:50 weight ratio retarded film dissolution by a factor of 2-3 compared to the PVP alone. In the third method, a novel approach was employed by placing drops of PAA solution on PVP drops on paraffin film and leaving to physically mix and dry down. In this proof-of-concept study, the washing-off profiles of the dry deposits revealed a striking rainfastness increase almost to the level of the insoluble controls. Methods employed in this study to increase rainfastness of agrochemical formulations can explain the previously reported effects of water-soluble polymers on rainfastness and allows the identification of improved rainfastness aids.

Keywords: Poly(N-vinyl pyrrolidone); rainfastness, cohesion; adhesion; agrochemicals; interpolymer complexes.

\section{Introduction}

Rain is one of the most important weather factors that adversely affect the performance of agrochemical compounds [1]. The extent of agrochemical rain wash-off is pertinent to the formulation, length of weathering and inherent tenacity of actives [2]. During rainfall as much as $90 \%$ of the initial agrochemical deposit applied on plants can be washed-off. This can reduce the 
effectiveness of applied crop protection agents and may lead to a requirement to reapply [1]. The environmental, economic and agronomic impact of the extra applications and off target movement of the active ingredient can be avoided by achieving improved rainfastness on leaves.

Specialized additives known as "stickers" are incorporated into the formulation or spray tank to address the issue of rain erosion [3]. Sticker adjuvants comprise polymeric materials or materials that autopolymerize and evaporate by air-drying leaving a flexible polymeric deposit on plant surfaces. This deposit protects agrochemical particles from rain washing and at the same time provides a controlled type delivery system thereby extending the residual activity of the active ingredient. The affinity-assisting properties of stickers for plant foliage are still poorly understood, but they can be tentatively described by various mechanisms like (1) surface tension reduction which assists droplet wetting and spreading; (2) physical retention by droplet infiltration into the plant stomata and surface microstructure; and (3) direct intermolecular adhesion via hydrophobic interactions, polar forces, electrostatic forces and hydrogen bonding [4-6]. In the past, simple sticker materials comprising fatty acids, mineral oils, glue, sugar, starches and natural resins were used, while more recently these have been replaced by latexes, resins and other synthetic polymers [7-12]. However, there is continuing need for new and more effective sticker adjuvants [13] as a lot of those currently used are associated with issues like formulation instability [11], locking up effects in pesticide biokinetics [10] and toxicological effects [8]. Yet, there is limited information on the fundamental principles governing the behavior of polymeric materials as rainfastness aids on plant surfaces.

There are three main methods to control the rainfastness of an agrochemical formulation using polymeric-based sticker adjuvants, i.e. using semi-crystalline thermoplastics with temperature controlling effects, ionic polymers with $\mathrm{pH}$-dependent dissolution and UV-polymerizable 
materials. Regarding the first two methods, previous studies $[14,15]$ have reported the effects of crystallinity, film dissolution and swelling degree of different grades of poly(vinyl alcohol) $(\mathrm{PVOH})$ and chitosan that underlie rainfastness behavior of agrochemical sprays on plant surfaces. On the basis of the mechanism, temperature and $\mathrm{pH}$ can significantly affect the hardening process of the rainfast deposit and upon evaporation, these materials solidify or form a water repellent gel on foliage which encloses the active ingredient. Latex-based adjuvants (e.g. Bond®) comprise colloidal dispersions of particles; as droplets dry down on the plant surfaces, latex particles pack together and eventually coalesce forming a continuous hydrophobic film [16]. Swelling and dissolution of the polymer film are retarded provided that its glass transition temperature $(\mathrm{Tg})$ is well above the ambient temperature in the field. Usually, there is a critical molecular weight of the polymer under which it is not rainfast. It has been concluded that polymers giving insoluble films, which can retard deposit erosion by water, gave enhanced rainfastness [14, 15]. Another type of controlled sticking mechanism is the in-situ UV-polymerization by which terpene materials are used, which polymerize when exposed to UV light [10]. However, the role of other factors must not be overlooked such as surface and adhesion phenomena between the polymer films, the plant surfaces and the plant topography.

Poly(N-vinyl pyrrolidone) (PVP) is a water-soluble polymer with a universal solubility in various solvents, high versatility and adhesive capacity to many surfaces; it can also tolerate high concentrations of electrolytes in solutions [17]. Different grades of PVP (soluble and insoluble) and copolymers of vinyl pyrrolidone with vinyl acetate (PVPVA) are commercially available with different molecular weights and exhibiting multifunctional properties, appropriate for numerous applications as binders, carriers, stabilizers, film forming and coating agents in both agrochemical and pharmaceutical fields. PVP has been claimed to be biodegradable and is biologically inert and 
exempt from the requirements of a tolerance concentration limits (EPA 40 CFR part 180.960) [17] and thus can be used in agri-food applications. Advantageously, in comparison to other polymers, PVP formulations improve residence and bioavailability of pesticidal agent by forming more homogenous and coherent deposits on the target surfaces $[9,12]$. However, to the best of our knowledge, there is no systematic study examining the correlation of the fundamental properties of PVP deposits, films, solutions and its rainfastness. As PVP has a moisture absorbing capacity, newer more hydrophobic copolymers of PVP have been introduced into the market to produce less hydrophilic materials with increased surface activity [18]. Alternatively, PVP solubility properties can be tailored by blending PVP with other polymers like polymonoethyl itaconate and polyacrylic acid (PAA) [19, 20]. In particular, PVP-PAA mixtures are known to form insoluble interpolymer complexes (IPC) due to hydrogen bonding [20]. Several authors indicated that these interactions are influenced by critical factors such as molecular weight, salt concentration, $\mathrm{pH}$, polymer concentration, solvent nature and polymer ratios [21, 22]. Nevertheless, there are no reports on the use of PVP-PAA blends or complexes as sticker adjuvants in agrochemical formulations.

This study addresses the evaluation of rainfastness activity of different grades of PVP in agrochemical formulations. A range of commercially available PVP grades were evaluated in detail by examining both their bulk polymer film properties and washing-off patterns using a range of lab-based established methods $[14,15]$ and theoretical models based on surface physicalchemistry. We also successfully demonstrated a new promising application technique for the sticker adjuvant inspired by the in situ polymer precipitation [23]. 


\section{Material and methods}

\subsection{Materials}

Different grades of PVP spanning various molecular weights (25-1300 kDa), vinyl acetate (VA) containing copolymers (PVPVA grades), with various solubilities and crosslinking degrees were chosen in this study (Table 1). Additionally, two grades of PVOH, one low (PVOH-L) and one high (PVOH-HM) molecular weights were used for comparison. All polymers were of analytical grade ( $\geq 98.0 \%)$. As a model agrochemical, the auto- fluorescent fungicide azoxystrobin (AZ) was provided by Syngenta (Jealott's Hill International Research Centre, Berkshire, UK) in the form of a 50\% mill base formulation containing xanthan gum as a rheological modifier. The commercial sticker adjuvant Bond ${ }^{\circledR}$ (De Sangosse Ltd) containing 45\% styrene-butadiene copolymer and 10\% alkoxylated alcohols was used as supplied throughout this study.

Table 1. Characteristics of polymers used in this study.

\begin{tabular}{|c|c|c|c|c|}
\hline Polymer & Supplier & Mw (kDa) & $\mathrm{M}_{\mathrm{w}} / \mathrm{M}_{\mathrm{n}}$ & Notes \\
\hline PVP40 & Sigma-Aldrich & 40 & $1.6 * *$ & \\
\hline PVP360 & Sigma-Aldrich & 360 & $\mathrm{ND}^{* * *}$ & \\
\hline PVP1300 & Sigma-Aldrich & 1300 & ND & \\
\hline Kollidon $25^{\circledR}$ & Sigma-Aldrich & Various & $\mathrm{NA}^{* * * *}$ & $\begin{array}{l}\text { Polydisperse } \\
\text { mixture of soluble } \\
\text { and insoluble } \\
\text { grades of PVP of } \\
\text { various Mw and } \\
\text { particle sizes. }\end{array}$ \\
\hline PVPVA13* & Sigma-Aldrich & 13 & ND & $\begin{array}{l}\sim 1: 2.4 \text { molar ratio } \\
\text { of VP:VA }\end{array}$ \\
\hline
\end{tabular}




\begin{tabular}{|l|l|l|l|l|}
\hline PVPVA50* & Sigma-Aldrich & 50 & ND & $\begin{array}{l}\sim 1.3: 1 \text { mole ratio } \\
\text { of VP:VA }\end{array}$ \\
\hline PVPP & Sigma-Aldrich & $\begin{array}{l}\sim 110 \mu \mathrm{m} \text { particle } \\
\text { size }\end{array}$ & NA & $\begin{array}{l}\text { Crosslinked PVP } \\
\text { (insoluble) }\end{array}$ \\
\hline PVOH-LM & Alfa Aesar & $21.7^{*}$ & $1.61^{* * * * *}$ & Fully hydrolized \\
\hline PVOH-HM & Alfa Aesar & $66.3^{*}$ & $1.54^{* * * * *}$ & Fully hydrolized \\
\hline PAA & Sigma-Aldrich & 450.00 & NA & $0.1 \%$ cross-linked \\
\hline
\end{tabular}

*VA indicates vinyl acetate in the copolymer. ** According to the information received from Merck European Technical Service; *** ND - not determined; **** NA - not applicable as the samples are weakly cross-linked; ***** Data taken from Symonds et al [15].

\subsection{Solution preparation}

Various stock solutions ( $2 \mathrm{wt} \%)$ of the polymers were prepared in deionized water $(\mathrm{pH} \simeq 7)$. All PVP samples except for the insoluble PVPP are water-soluble polymers having an almost universal solubility in many organic solvents. Here, the concentration used was low enough, so only a few hours of moderate stirring was enough for a complete dissolution of PVP. PVOH solutions instead required heating and stirring at $90{ }^{\circ} \mathrm{C}$ for around $1 \mathrm{~h}$ and further stirring overnight without heating. Additionally, PVP mixtures were prepared by mixing PVP and PAA aqueous solutions ( $2 \mathrm{wt} \%)$ at the stoichiometric weight ratios 75:25, 50:50 and 25:75. Due to the strong complexation between the two polymers at their critical $\mathrm{pH}$ value $\left(\mathrm{pH}_{\text {crit }} \leq 3.8-4.3\right.$; see Table 2$)$, the $\mathrm{pH}$ of the mixtures was adjusted to 5.0 by adding small amounts of $1.0 \mathrm{M}$ sodium hydroxide. This step was necessary to ensure uniform mixtures and homogenous application of formulations. 
Table 2. $\mathrm{pH}$ critical values $\left(\mathrm{pH}_{\text {crit }}\right)$ for PVP-PAA and PVPVA-PAA blends at different stoichiometric weight ratios $\left(\Phi_{\mathrm{w}}\right)$. Critical $\mathrm{pH}$ values correspond to the $\mathrm{pH}$ were the turbidity $(\lambda=$ $400 \mathrm{~nm})$ is increased abruptly in the turbidity-pH plot of each mixture. $\mathrm{M}_{\mathrm{w}}(\mathrm{PVP})=1.300 \mathrm{kDa}$. $\mathrm{M}_{\mathrm{w}}(\mathrm{PAA})=450 \mathrm{kDa}$. Concentration of polymers $=2 \% \mathrm{wt}$.

\begin{tabular}{|l|l|l|}
\hline $\boldsymbol{\Phi}_{\mathbf{w}}$ & $\mathbf{p H}_{\text {crit(}}($ PVP-PAA) & pH $\mathbf{H r i t}^{(\text {PVPVA-PAA) }}$ \\
\hline $75: 25$ & 3.84 & 4.17 \\
\hline $50: 50$ & 3.85 & 4.37 \\
\hline $25: 75$ & 4.10 & 4.25 \\
\hline
\end{tabular}

\subsection{Swelling-dissolution of films}

Cast films of different polymer grades were prepared by pouring each polymeric solution into petri dishes and placing them in a horizontal position for 5 days to dry. Dissolution behavior of films was evaluated by putting small samples into a nylon mesh bag and immersing into a $100 \mathrm{~mL}$ beaker

of deionized water equilibrated at $25^{\circ} \mathrm{C}$. The mesh bag with the film was weighed at different time intervals and the mass of each sample remaining in the bag was estimated. The swelling degree (SD) or hydration of the films was calculated according to equation (1):

$\mathrm{SD}=\frac{\mathrm{m}-\mathrm{m}_{0}}{\mathrm{~m}_{0}}$

where $\mathrm{m}$ is the mass of the film at time $\mathrm{t}$ and $\mathrm{m}_{0}$ the mass of the dry film. 


\subsection{Work of adhesion and affinity}

Surface tension. The static surface tension (Table S1) for different polymer formulations (0.4 wt \%) was estimated by the capillary rinse method [24] using equation (2):

$\gamma=\operatorname{pgh} \frac{\mathrm{R}_{\mathrm{x}^{2}}}{2 \mathrm{R}_{\mathrm{y}^{2}}},\left(\mathrm{mN} \mathrm{m}^{-1}\right)$

where $\mathrm{p}$ is the density of polymer solutions, $\mathrm{g}$ is the acceleration due to gravity, $\mathrm{h}$ is the height of solution rise into the glass tube (internal radius $0.9 \mathrm{~mm}$ ) measured between the surface of the solution and the bottom of the meniscus inside the tube, and $\mathrm{R}_{\mathrm{x}}, \mathrm{R}_{\mathrm{y}}$ are the equatorial and axial radii of the ellipsoidal curved surface of the meniscus inside the tube, as calculated using ImageJ software. Photomicrographs were taken with the Conrad DP-M17 USB digital microscope (Conrad Electronics, Hirschau, Germany). We assumed the total wetting of aqueous based solutions (neglecting the contact angle effects) for the hydrophilic glass capillaries used in this test.

Contact angle measurements. Drop volumes $(5 \mu \mathrm{L})$ of each formulation were placed (15 drops per sample) on parafilm surfaces fixed on glass slides by double-side sticky tape. The equilibrium advancing contact angles $(\theta)$, formed at the junction of solid-liquid-vapour phases, were measured by the static sessile drop method using the Attention Theta Lite goniometer (Bioline Scientific, Sweden) at room temperature.

Work of adhesion. The total work of adhesion $\left(\mathrm{Wa}, \mathrm{mJ} \mathrm{m}^{-2}\right)$, the energy needed to separate the drop from the surface, was determined from the combined Young- Dupré equation for liquid dropon solid surface (3): 
$\mathrm{Wa}=\gamma_{\mathrm{s}}+\gamma_{1}-\gamma_{\mathrm{sl}}=\gamma_{1}(1+\cos \theta)$

where $\gamma \mathrm{s}, \gamma \mathrm{l}$ and $\gamma \mathrm{sl}$ the surface tension of solid surface, the surface tension of polymer solution and the interfacial tension between the solid surface-drop interface, respectively. Since surface energies do not alter profoundly when the drop solidifies, we assumed in this work that apart from any shrinkage-related stress from liquid to solid, the Young-Dupre equation also describes a situation applicable to solid deposits- on- solid substrates.

Additionally, the affinity $(\Delta \delta)$ of the polymers for the paraffin film surfaces was calculated [5] by matching their 3-D solubility parameters (see Supporting Information; Estimation of affinity of polymers for paraffin film surfaces, Table S2 and Table S3).

\subsection{Viscosity}

Viscosity measurements were taken with a Brookfield viscometer DV-II+ Pro (Brookfield Engineering Laboratories Inc, USA) by pouring the polymer solutions $(0.4 \% \mathrm{wt})$ into a $200 \mathrm{~mL}$ glass beaker and registering the viscosity with a \#S3 spindle at different shear rates (20-100 rpm) beginning with the highest rate and then measuring it in a increasing order [25]. The temperature of the samples during the measurements was kept constant at $20-20.5{ }^{\circ} \mathrm{C}$. The viscosity of the blends at different blend ratios was expressed as gain of viscosity [26] (4):

$g=\frac{\eta_{\text {mixture }}}{\eta_{P V P+\eta_{P A A}}}$,

where $g$ is the viscosity gain, $\eta_{\text {mixture }}$ the viscosity of the blend, $\eta_{P V P}$ the viscosity of PVP solution and $\eta_{\text {PAA }}$ the viscosity of PAA. The intrinsic viscosity, $[\eta]$, of polymers was determined by extrapolation the relative viscosities of a series of solutions of different concentrations to zero 
concentration. The reciprocal of $[\eta]$ was used as a first approximation to represent the overlap concentration $\left(\mathrm{c}^{*}\right.$, in $\left.\% \mathrm{wt}\right)$ of the flexible polymer solution in water [27]:

$c^{*}=\frac{1}{[\eta]}$

\subsection{Thermal Analysis}

Residual moisture content ( $\mathrm{wt} \%$ of the sample mass) of films was measured using thermal gravimetric analysis (TGA,TA Q50, TA Instruments, USA) carried out in the temperature range from 50 to $200{ }^{\circ} \mathrm{C}$ at a rate of $10{ }^{\circ} \mathrm{C} \mathrm{min}^{-1}$ in a nitrogen atmosphere.

Glass transition temperature ( $\mathrm{Tg}$ ) was determined using differential scanning calorimetry (DSC,TA Q2000 instrument, TA Instruments, USA) performed at a temperature range between 25 to $200{ }^{\circ} \mathrm{C}$ with a heating rate of $10{ }^{\circ} \mathrm{C} \cdot \mathrm{min}^{-1}$ in an argon atmosphere. Determination of $\mathrm{Tg}$ was followed by analyzing DSC thermograms using the Advantage TA Universal Analysis software available online from TA instruments.

\subsection{Scanning electron microscopy (SEM)}

SEM examination of the topography of dry deposits on paraffin film was performed by cutting small samples $\left(0.5 \mathrm{~cm}^{2}\right)$ with an acetone-cleaned razor blade and mounting on aluminium stubs with an adhesive carbon tab. For the examination of the adhesive interface between deposits and paraffin film, samples were thick sectioned with a razor blade and fixed on a vertical position on stubs by dipping the lower side of the section in an Alardite ${ }^{\circledR}$ epoxy resin mixture. Next, mounted samples were placed on a diode sputter device for gold coating in an argon atmosphere. SEM examination of the samples was followed by using the FEI Quanta FEG 600 Environmental Scanning Electron Microscope with an acceleration voltage of $20 \mathrm{kV}$. 


\subsection{Rainfastness activity}

Drops $(10 \mu \mathrm{L})$ from different formulations containing $0.4 \mathrm{wt} \%$ of the polymer and $1 \% \mathrm{w} / \mathrm{v} \mathrm{AZ}$ were placed by microliter syringe on model (paraffin film) and plant surfaces (Vicia faba leaves growth stage 18, GS18 [28]) fixed on horizontal plastic boards. Plants were provided by Syngenta Ltd and their growth conditions are described elsewhere [15]. The insoluble PVP grade crospovidone (PVPP) was applied by direct mixing small amounts of powder into the test solution following vigorous stirring for $2 \mathrm{hr}$. Drops were left to evaporate at $25{ }^{\circ} \mathrm{C}$ for $1.5 \mathrm{~h}$ (unless otherwise stated) and successively washed-off with $1 \mathrm{~mL}$ of deionized water at a rate of $2 \mathrm{~mL} \cdot \mathrm{min}^{-}$ ${ }^{1}$ simulating about $12 \mathrm{~mm}$ per hour rain. Rainfastness was estimated by employing the fungicide azoxystrobin, which has been used previously [14] as a model fungicide due to its fluorescent properties. In that method, the residual fluorescent activity of the dry deposits after each successive wash was measured with fluorescent microscopy (Leica MZ10F). Rainfastness was expressed as the adjusted coverage \% (fluorescent intensity after each washing step normalised to a starting intensity) of the initial fluorescent activity before the washing procedure using ImageJ software. All fluorescent images were taken under the same settings with the excitation and emission wavelengths of $490 \mathrm{~nm}$ and $537 \mathrm{~nm}$, respectively.

\subsection{Drop-on-drop method}

This method is essentially described above. However, each polymer component (PVP or PAA) were instead placed separately ( $5 \mu \mathrm{L}$ drop of each polymer solution) by allowing a drop of PAA 
solution to impinge onto another drop of PVP resting on the underlying surface. Careful consideration was given to leave droplets to physically coalesce without any further mixing.

\subsection{Statistical Analysis}

Within each washing time, rainfastness of different grades of PVP and its PAA blends relative to the controls (Bond, $\mathrm{PVOH}$ ) were analyzed by using the Dunnett's post hoc test as part of the oneway analysis of variance (ANOVA). The influence of the different ratios of PVP-PAA blends on the rainfastness were analyzed with the one-way ANOVA Tukey's post hoc test. Evaluation of rainfastness data for the drop-on-drop method was performed by running the independent-samples

T-test. Swelling dissolution data were analyzed by performing two-way ANOVA with Tukey's post hoc test in order to detect any significant differences (at $95 \%$ level) between the dissolution time-courses for different polymers ratios. All the analyses were run using SPSS 21.0 (IBM, USA).

\section{Results and discussion}

A range of PVP properties as related to agrochemical rainfastness on both model and plant surfaces were evaluated using fluorescence microscopy (FM), scanning electron microscopy (SEM), thermal analysis, viscosity, surface analysis and dissolution tests. Table S4 provides information about different physicochemical properties of PVP cast films used in swelling-dissolution studies and the aqueous solutions used for film preparations. Generally, there was dependence between 
the molecular weight and the viscosity, residual moisture content, surface tension and dissolution behavior of PVP formulations.

\subsection{Viscosity}

Viscosity was increased with increasing the molecular weight of PVP (Table S4), a result normally expected according to the Flory and Fox theory of dilute or semi-dilute polymer solutions [29]. The estimated overlapping concentration $\left(\mathrm{c}^{*}\right)$ of different PVP polymer solutions was varied between 0.12 to $0.17 \% \mathrm{wt}$, which is 2.3 - to 3.3 -times smaller than $\mathrm{c}_{\text {polym }}$ used $(0.4 \% \mathrm{wt})$. This indicates that polymer solutions were in semi-dilute region $\left(\mathrm{c}^{*}<\mathrm{c}\right)$ and some entanglement formation of polymer coils may be present. PVP-PAA mixture solutions gave a characteristic positively deviating behavior (Figure 1), which is common for systems forming interpolymer complexes. This is for example with polymer associations formed between poly(carboxylic acids) and non-ionic polymers via hydrogen bonding [26].

The viscosity gain was plotted against PVP or PVPVA ratio in the mixture and it was shown that with increasing PAA content the viscosity increased. The PVP-PAA mixture showed a greater gain in viscosity than PVPVA-PAA, especially at the 50:50 weight ratio. These observations have been explained by the loss of free volume demonstrating the miscible character for the blends [29]. Also, the hydrodynamic character of PAA in the mixture contributes to that deviating behavior. It was revealed by DLS analysis that PAA in the aqueous solution behaves as unfolded coil chains stretching around 30 times more in length than PVP imparting a lower diffusion coefficient (Figure S1). 


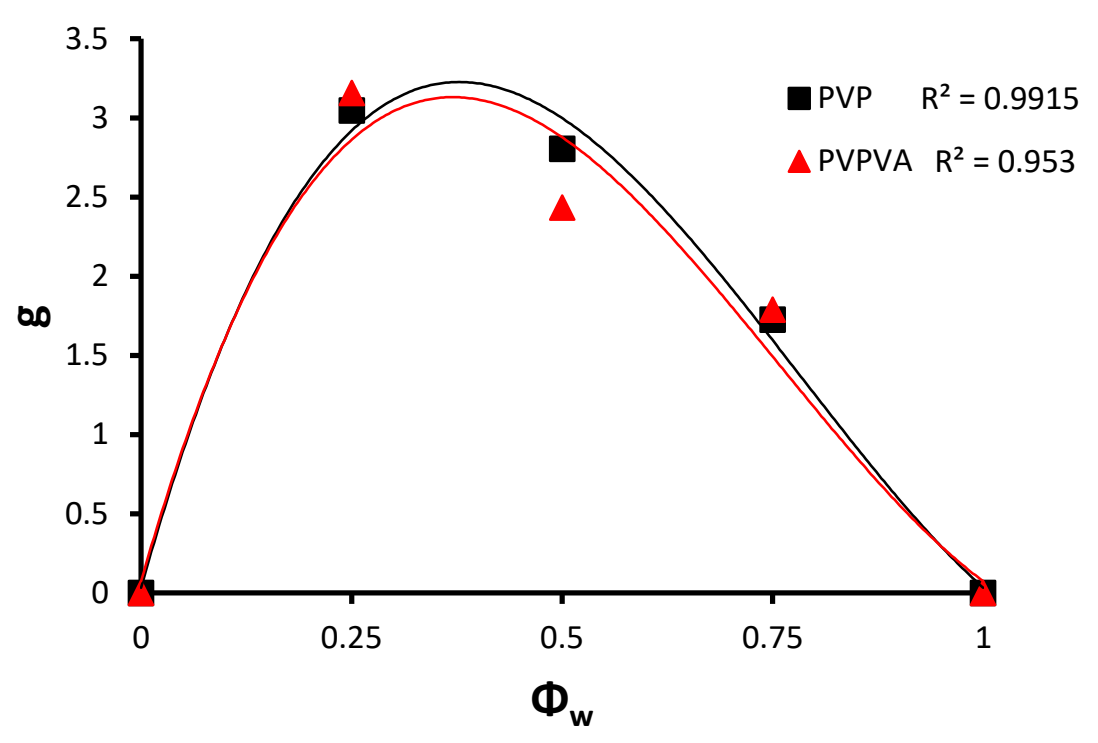

Fig. 1. Viscosity gain dependence on stoichiometric weight ratio ( $\Phi w)$ of PVP or PVPVA in PVPPAA and PVPVA-PAA systems at $20.9^{\circ} \mathrm{C}$. $\mathrm{C}_{\text {polymers }}=0.4 \%$ wt. Error bars are not displayed as these were very small. The lines are calculated from the least squares fitting method of the equation 4 .

\subsection{Swelling-dissolution of films}

Rain can have a dramatic impact on the residual life of dry agrochemical deposit. The latter is translated into how easy water molecules can separate polymer coils from each other (cohesive interactions) and erode the polymer film which entraps the active ingredient. Swelling-dissolution profiles of polymeric films can give a direct indication about the rain erosion of the formulation deposits $[14,15]$.

Results showed that all grades of PVP dissolved within 1 min after immersion in a water bath (Figure 2). The dissolution rate was decreased with increased PVP molecular weight, in accordance with our previous reports with $\mathrm{PVOH}$ and chitosan $[14,15]$. The lowest molecular weight grades PVP40 and PVPVA50 dissolved within the first $10 \mathrm{sec}$ rendering their further 
analysis impossible. Besides the molecular weight, the polydispersity affects the dissolution rate [30] as found here with more polydisperse Kollidone $25 \AA$. Moreover, the higher the Mw, the higher the maximum swelling capacity with PVP1300 kDa reaching a maximum swelling degree almost 2 times higher than lower $\mathrm{Mw}$ grades. This is explained by the greater extent of entanglement in larger molecular weight grades which can yield higher film expansion [30].

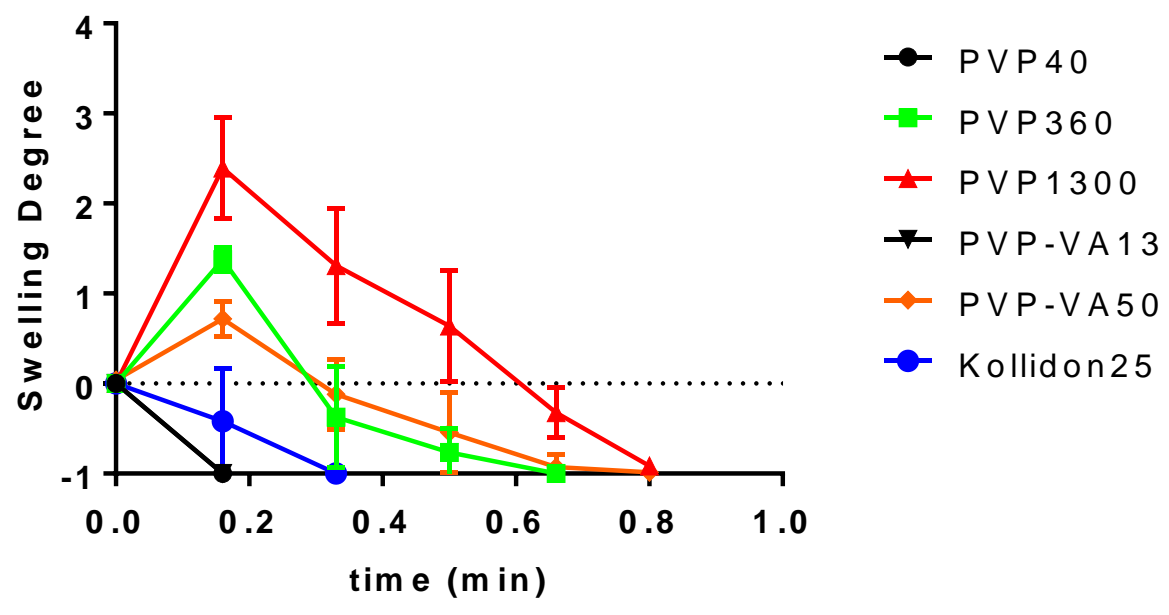

Fig. 2. Swelling and dissolution kinetics of PVP films after immersion in deionized water at 25 ${ }^{\circ} \mathrm{C}$. Films were prepared by casting $2 \% \mathrm{w} / \mathrm{v}$ PVP solutions and allowing to evaporate for 5 days. Results are expressed as the mean value $(n=4) \pm$ standard deviation, where positive values (above the dashed line) mean swelling and negative values (below the dashed line) mean dissolution.

Nonetheless, the extent of PVP dissolution was significantly higher $(\mathrm{p}<0.05$, Tukey's post hoc test $)$ in relation to that reported to the fully hydrolyzed $\mathrm{PVOH}(\mathrm{Mw}=90 \mathrm{kDa})$, which showed that even after 1-day immersion in water bath PVOH films remain almost undissolved [15]. It was suggested previously [15] that semi-crystalline nature of PVOH is likely associated with the slow polymer hydration. 
It has been reported that PVP can form strong interpolymer complexes with poly(acrylic acid) (PAA) [21, 31- 33]. Hydrogen bonding is the primary interaction mechanism between these two polymers and the extent of the complex formation is a function of solution $\mathrm{pH}$. Specifically, the formation of insoluble complexes is observed below a certain critical $\mathrm{pH}$ [21]. In this study it was found that $\mathrm{pH}_{\text {crit }}$ (Table 2) was dependent on the type of PVP rather than the mixing ratio of the polymers with the PVPVA exhibiting stronger complexation ability than PVP. This can be related to less polar vinyl acetate groups of PVPVA, which contribute to hydrophobic stabilization of interpolymer complexes.

The swelling-dissolution of films was investigated as a function of a polymer weight ratio in the PVP-PAA mixtures. As seen from Figure 3A the blends exhibited retarded dissolution compared to PVP alone. For PVP-VA copolymer (Figure 3B) the dissolution was almost two times faster than that of PVP-PAA mixture despite their higher complexation activity compared to PVP-PAA. This observation can be due to the better thermodynamic compatibility of copolymer with PAA as the vinyl acetate groups of the copolymer may cause greater free volume expansion of the film. This hypothesis supports the film thickness measurements for mixtures (Table S4) suggesting that some mixtures are less dense for a given mass per unit area (having a higher moisture content) and thus they might exhibit a faster imbibition of water into their deposits. Moreover, the polymer weight ratio of mixtures affected the dissolution rate with the 50:50 giving the lowest rate. DLS studies [34] reveal that at this ratio interpolymer complexes are strongly associated with a more compact structure. 


\subsection{Thermal analysis of films}

Residual water content (Table S4) was estimated thermogravimetrically and it was found to be around 1.5-12.4 wt \% of film depending on the molecular weight and the PVP-PAA mixing ratio. Within the different blends the 50:50 ratio retained the highest percentage of water. The residual water component in the film may have an influence on the bioadhesion by expanding the adhesive material and increasing the flexibility and mobility of the polymer chains on the underlying surface [35].

A

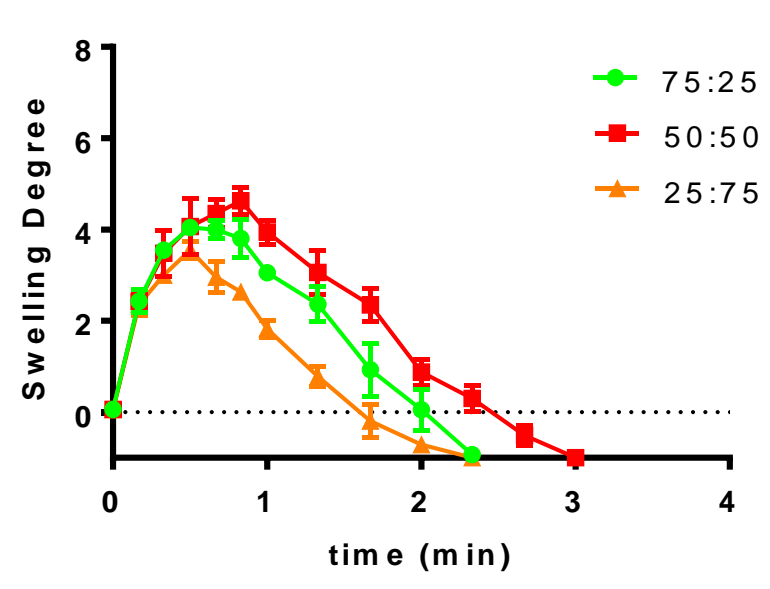

B

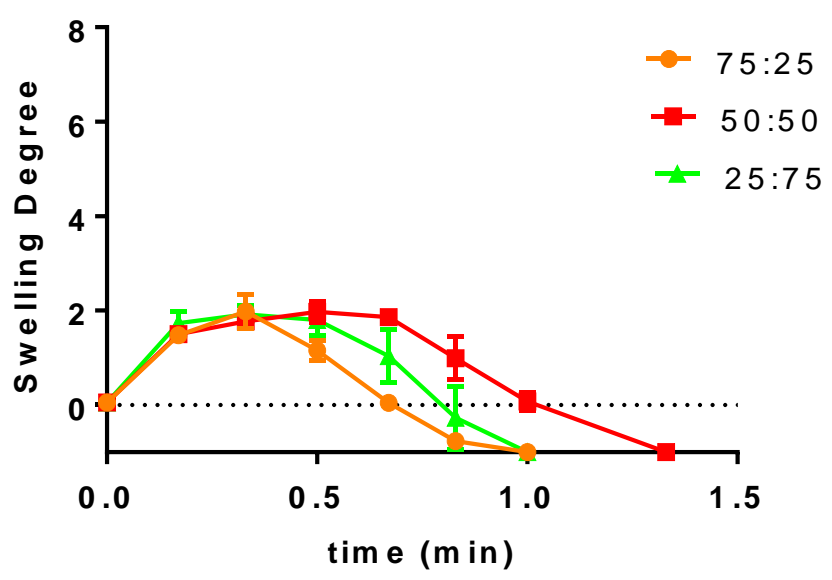

Fig. 3. Dissolution kinetics of PVP-PAA (A) and PVPVA-PAA (B) films after immersion in deionized water at $25{ }^{\circ} \mathrm{C}$. Films were prepared by casting $2 \% \mathrm{w} / \mathrm{v}$ mixture polymer solutions of different stoichiometric weight ratios $(75: 25,50: 50$ and $25: 75)$ and allowing to evaporate for 5 days. Results are expressed as the mean value $(n=4) \pm$ standard deviation, where positive values (above the dashed line) mean swelling and negative values (below dashed line) mean dissolution. 
All mixtures met the miscibility criterion for a single Tg appearance [36], which was around the Tg of PAA as confirmed by DSC analysis (Table 3). None of the samples showed any measurable Tg at around $102{ }^{\circ} \mathrm{C}$, where it is assigned to Tg of PVP [37]. The glass transition temperature determined for pure PAA film $\left(56.7^{\circ} \mathrm{C}\right)$ is in disagreement with the literature data for dry polymer but agrees well with the data found for PAA films containing some moisture [38, 39]. The films used in our work showed to have $5-10 \% \mathrm{w} / \mathrm{w}$ (data not shown) residual moisture which could explain this deviation in Tg. For PVP-PAA mixtures and especially for the 50:50 blend a striking lower enthalpic change $(\Delta \mathrm{H})$ was associated with the Tg in comparison with PVPVP-PAA (Table 3).

Table 3. DSC thermal characteristics of PVP-PAA and PVPVA-PAA blends.

\begin{tabular}{|l|l|l|l|}
\hline Sample & $\Phi \mathrm{W}\left(\mathrm{w} \mathrm{w}^{-1}\right)^{*}$ & $\mathrm{~T}_{\mathrm{g}}\left({ }^{\circ} \mathrm{C}\right)$ & $\Delta \mathrm{H}\left(\mathrm{J} \mathrm{g}^{-1}\right)$ \\
\hline PVP1300 & $100: 00$ & 102.3 & 172.7 \\
\hline PVPVA50 & $100: 00$ & 49.58 & 6.10 \\
\hline PAA450 & $100: 00$ & 56.7 & 6.04 \\
\hline PVP-PAA (a) & $75: 25$ & 47.43 & 0.67 \\
\hline PVP-PAA (b) & $50: 50$ & 58.35 & 0.16 \\
\hline PVP-PAA (c) & $25: 75$ & 49.64 & 3.27 \\
\hline PVPVA-PAA (a) & $75: 25$ & 53.6 & 4.67 \\
\hline PVPVA-PAA (b) & $50: 50$ & 57.75 & 3.62 \\
\hline PVPVA-PAA (c) & $25: 75$ & 54.94 & 3.04 \\
\hline
\end{tabular}

*Mixture of two polymers in films based on their weight ratio $\left(\Phi_{\mathrm{w}}\right)$.

The lower $\Delta \mathrm{H}$ postulates enhanced physicochemical properties of PVP-PAA samples because of the lower flexibility of the polymer chains due to the strengthened polymer complexation [40], in 
connection with the above data on viscosity and dissolution tests. Supplementary data (Figure S210) also indicated that all mixtures, except for PVP-PAA 25:75 were stable in the full range of compositions and temperatures tested. The PVP-PAA 25:75 mixture showed some thermal features in DSC thermogram above $127{ }^{\circ} \mathrm{C}$ possibly due to decomposition as confirmed by the blob-like appearance of the sample when the pan visually examined after the thermal process. Noticeably, a sharp endothermic peak for all blends was detected at around $125-145{ }^{\circ} \mathrm{C}$ corresponding probably to the melting point $\left(\mathrm{T}_{\mathrm{m}}\right)$ of $\mathrm{NaOH}$ traces present in the films (Figure $\mathrm{S} 5$, S7-10).

\subsection{Rainfastness}

The rainfastness of PVP formulations was quantified by monitoring the loss of the AZ particles from paraffin film and leaf surfaces with fluorescence microscopy after a number (1-10) of successive washing steps. It was advantageous to use a paraffin film as a model plant surface since it has a chemically defined composition, which simplifies the quantification of microscopic measurements.

The wash-off profile of different grades of PVP on paraffin film surfaces is shown in Figure 4. Results show that among the different PVP grades (Figure 4B) only the highest molecular weights $360 \mathrm{kDa}$ and $1300 \mathrm{kDa}$ increased the residence of $\mathrm{AZ}$ by up to 3 times compared to control. This rainfastness, however, was still significantly ( $<<0.05$, Dunnett's post hoc test) lower than the commercial adjuvant Bond and $\mathrm{PVOH}$, which showed greater retention even after up to 10 washes. The other grades (lower Mw, polydisperse Kollidone 25®, vinyl acetate copolymers and the crosslinked PVPP) were washed-off within 1-2 washing cycles. This seems to indicate a molecular weight dependence of rainfastness (Figure 4C) as has been reported with many other ionic and 
non-ionic polymers such as PVOH, chitosan, cellulose-based polymers, polyethylene oxide, and polyterpenes $[9,10,14,15]$.

Nonetheless, it seems that within a homologous series of a polymer besides the existence of a threshold molecular weight [15] an upper limit of molecular weight also exists over which rainfastness activity does not increase further. Over this limit, the washing-off is influenced by the inherent chemical properties of the employed polymer. Although a critical molecular weight (Mc) may be necessary for entanglements to occur at the polymer-paraffin film interface especially for polymers with $\mathrm{Mw}>\mathrm{Mc}$, other factors may also be important such as linear chain (coiled) conformations at very high $\mathrm{Mw}(\mathrm{Mw}>>\mathrm{Mc})$. In particular, the most rainfast grades PVP360 and PVP1300) have also molecular weights many times greater than the Mc of PVP $\left(\mathrm{Mc} \approx 29 \cdot 10^{3}\right.$, see Supplementary Information). It can also be observed that as compared to other polymers, the latexbased adjuvant Bond is more susceptible to rain washing during the initial washing events. This can be possibly attributed to the way fungicide particles were dispersed after latex film formation. Closer fluorescence microscopic examination of the deposits (Figure S11) reveals that rain washing removes only the fungicide particles on the deposit surface directly exposed to water, while a significant fraction of the fungicide particles remained strongly bound to the film subsurface and were unsusceptible to rain washing. 

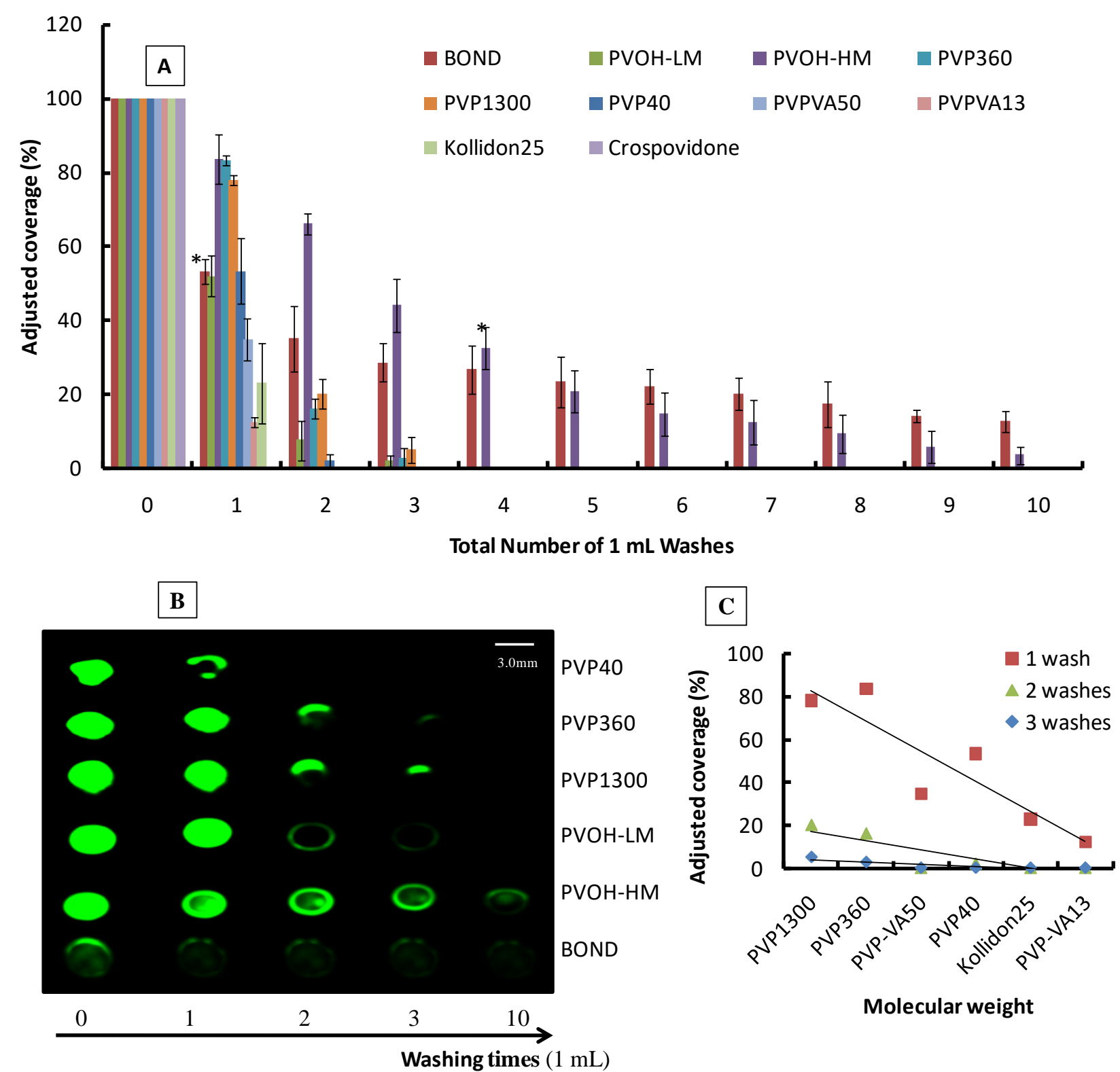

Molecular weight

Fig. 4. A. Wash-off profiles of the different polymer samples on paraffin film surfaces. Measurements are presented as the mean $(\mathrm{n}=3)$ fluorescent spread area \pm standard deviation of the deposited AZ particles after being processed with ImageJ. * No significant difference ( $p>0.05$, Dunnett's post hoc test). B. Representative FM images of PVP samples and the controls (PVOH and Bond) showing their washing-off pattern during simulated rain washes. C. Relationship between the molecular weight and the rainfastness of PVP samples after 1, 2 and 3 washes. 
Figure 4B shows representative fluorescent images of the deposits during the washing procedure. Distinct deposit patterns appear depending on the type and the molecular weight of the polymer. In the case with both controls (Bond and PVOH), the deposits show the characteristic coffee-ring pattern common in adjuvants exhibiting high surface activity. For Bond, this activity may arise from the surfactants present in the formulation, while PVOH is a polymeric surfactant itself [40]. Surface tension measurements (Table S4) confirm that PVP does not significantly reduce the surface tension of the solution, while $\mathrm{PVOH}$ and especially its higher molecular weight grade can reduce the surface tension by around $68 \%$ compared to that of deionized water. In the case of PVP, the ring effect was suppressed and fungicide particles have been homogenously deposited as a coherent deposit, which ensures the good film forming properties of the polymer in the AZ formulation. Also, as can be seen, during the washing time course experiment, the PVP deposit erosion is centripetal, while for PVOH erosion proceeds outwards.

It can be seen that with PVP and PVOH the film swelling is a rate limiting step in rainfastness as compared to the non-swollen polymer (Bond). Visual examination of the deposits with microscope during the rain-washing test showed that within the first washing times where there is a minimum AZ loss (Fig. 4A) the deposits also show a maximum volume expansion without any associated erosion. We assumed that a greater and slower swelling stage can delay the film dissolution and thus providing more tenacious deposits against rainwater. Additional evidence to this hypothesis comes from a dissolution - AZ release test (Figure 5). The latter shows that fast AZ release profile from polymeric deposits co-exists with the onset of fast dissolution of PVP polymer. 


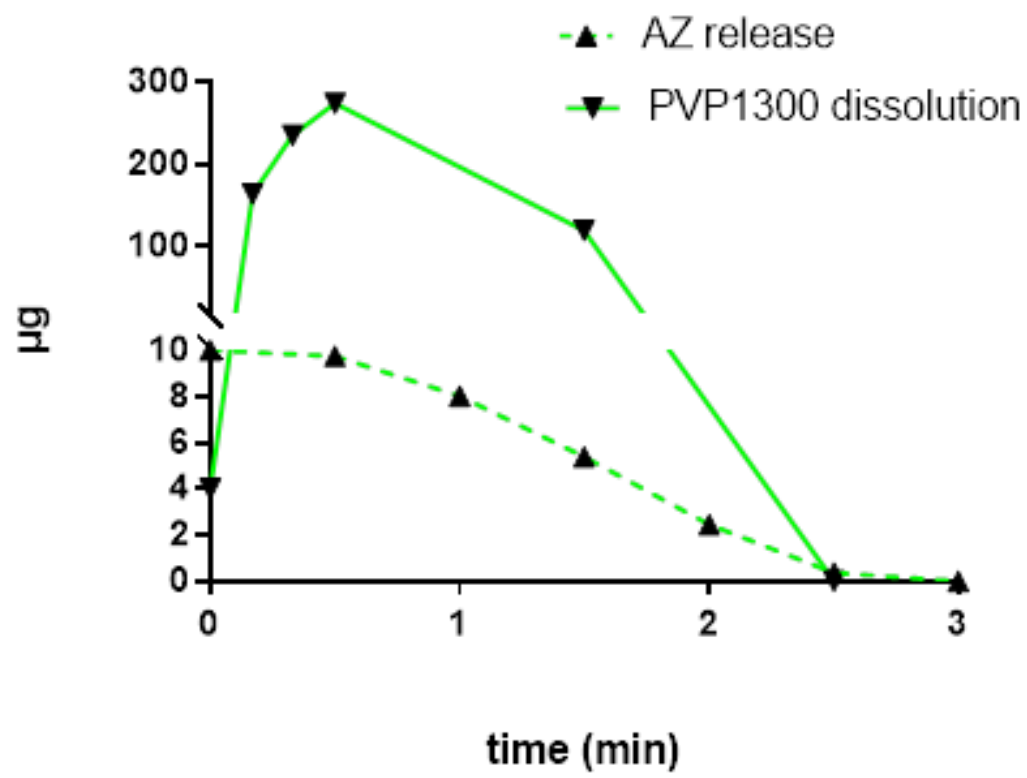

Fig. 5. AZ mass release from PVP deposits (measured from fluorescence intensity) on paraffin film surface during the washing test vs the mass evolution of PVP from the dissolution test.

To give a quantitative treatment of the rainfastness behavior as related to adhesive interactions, the work of adhesion from different PVP formulations was calculated by means of surface activity and contact angle measurements (Figure 6A). A clear relationship between the work of adhesion and rainfastness was observed but only within the homologous series of different polymer molecular grades. The Young's-Laplace equation (Eq. 3) predicts that high surface tension and interfacial tension are required for strong adhesion [41] and this was validated only for PVP grades, while PVOH showed that the stronger adhesion was associated with PVOH-HM, exhibiting lowest surface tension (Supplementary information) in comparison with PVOH-LM. 


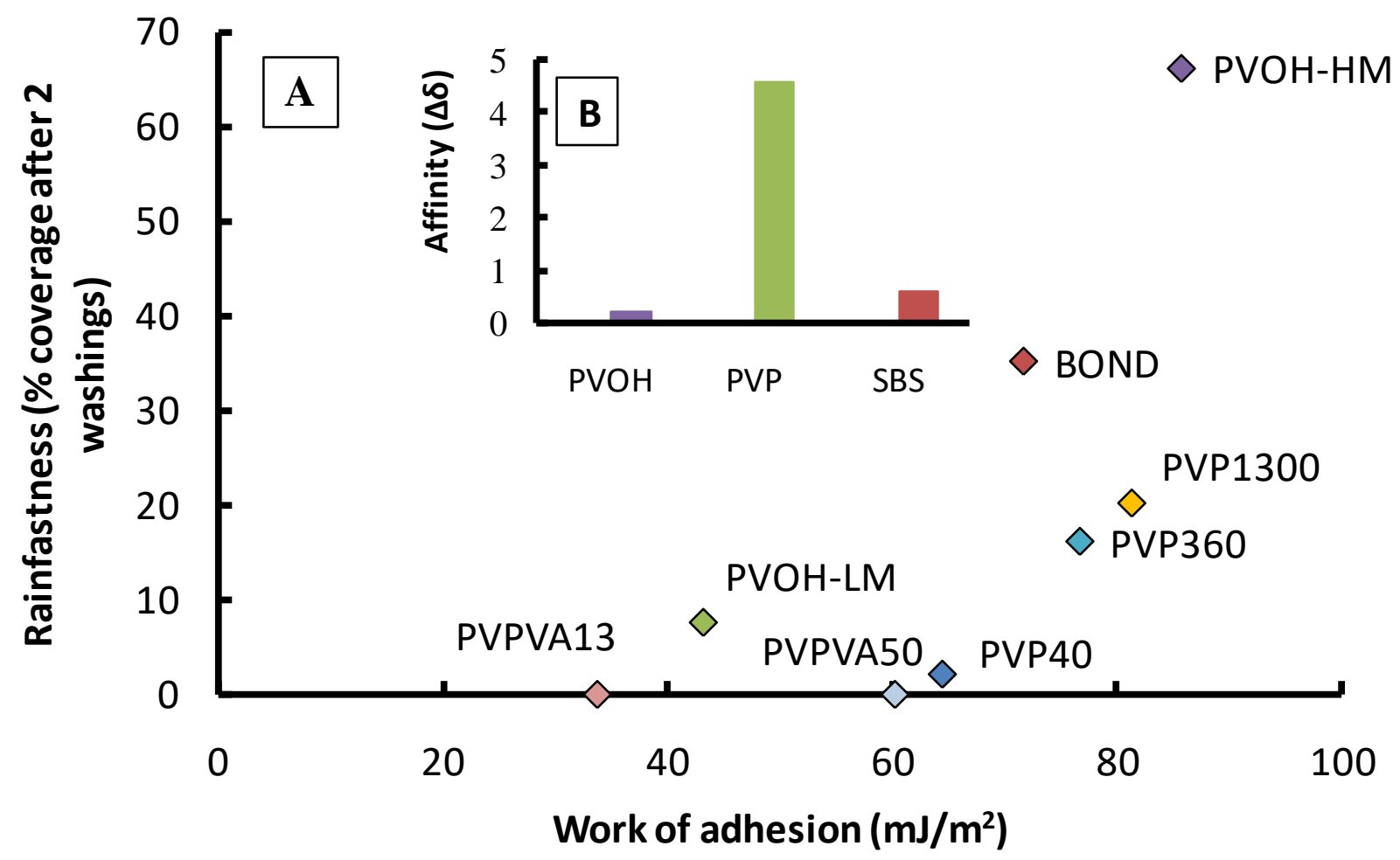

Fig. 6. A. Relationship between work of adhesion and rainfastness (after 2 washes) of tested liquid formulations. B. Affinity of polymers used for paraffin film surface (Supporting Information). The prediction of the affinity $(\Delta \delta)$ of the commercial adjuvant Bond was based on styrenebutadiene block polymer (SBS) which is the active component of the product (see eq. S3 and Table S3).

While it is indicated that the molecular weight of polymers plays an important role in the rainfastness, possibly by increasing the polymer-adsorbed fraction (physisorption), more important seems to be the hydrophobic-lipophilic nature of the polymer. Both paraffin film and plant membranes are characterized as lipophilic due to the presence of lipids and hydrocarbons in their structure and thus it is expected that polymers with higher dispersion forces will exhibit greater affinity for that surfaces [5]. We found that the affinity of different polymers to model 
surfaces (Figure 6B) was decreased in the order of $\mathrm{PVOH}>\mathrm{Bond}>>\mathrm{PVP}$, partially explaining the weak rainfastness of PVP in comparison with the controls.

The faster dissolution rate of films possibly explained their weak rainfastness activity of PVP formulations as compared to the controls leading us to proceed with the idea of exploring the interpolymer complexation approach for retarding polymer dissolution and extending the residual AZ life under wash off. This hypothesis was tested only with the highest molecular weight PVP1300 and its copolymer PVPVA50 as components in the mixtures with PAA. The effect of PVP complexation with PAA on rainfastness was tested by two methods, namely a) pre-blended PVP-PAA mixtures and b) drop-on-drop method.

Premixed PVP-PAA. Rainfastness of deposits (Figure 7) was significantly enhanced ( $<<0.05$, Dunnett's post hoc test) for all mixtures by 3 times in comparison with PVP or PVPP alone. Among the two types of blends, PVP-PAA mixtures were more rainfast with 50:50 ratio giving the highest residual activity over the washing events, which agrees with the previous swelling-dissolution data of their films. Interestingly, mixtures with higher PAA rendered deposits less sticky $(\mathrm{p}<0.05$, Tukey's test) and after about 3 washings deposits have shown to delaminate from paraffin film surface rather than dissolve. This phenomenon was more pronounced in PVP-PAA mixtures. This suggests that there is an optimum ratio of the two polymers for maximum rainfastness regardless of the degree of complexation. Possibly, as these mixtures containing higher fractions of PAA had also a higher amount of $\mathrm{NaOH}$ to prepare them thus their deposits may dissolve easily due to the solubilizing effect of the salt. Future research into the behavior of interpolymer complexes in rinse water is needed to investigate the poor adhesion of PVP-PAA deposits which showed delamination in previous tests. 

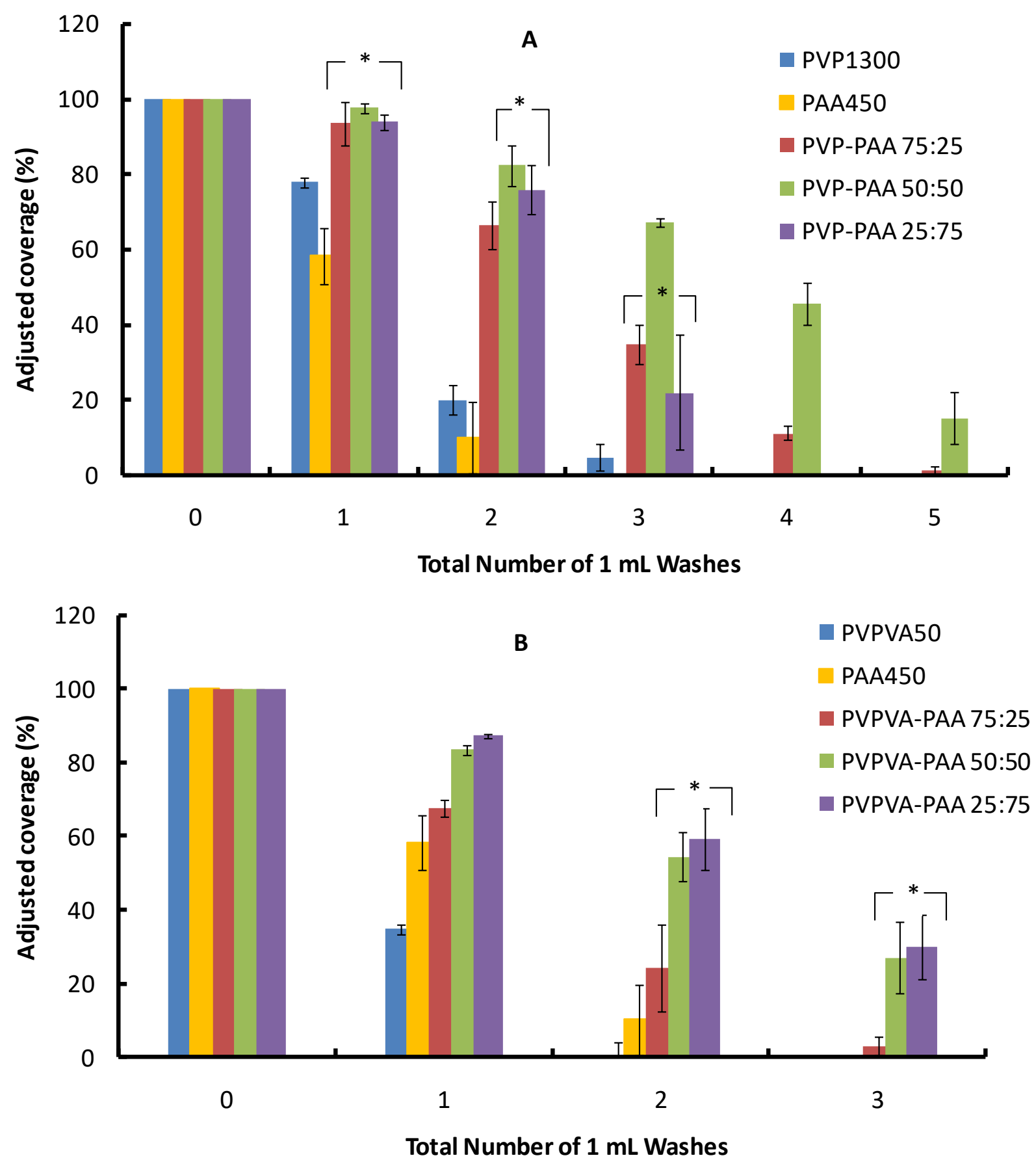

Fig. 7. A. Wash-off of PVP-PAA (A) and PVPVA-PAA (B) mixtures on paraffin film surfaces. Measurements are presented as the mean $(\mathrm{n}=3)$ fluorescent spread area \pm standard deviation of the deposited AZ particles after processed with ImageJ. * No significant difference ( $p>0.05$, Tukey's test). Mixture samples refer to PVP1300, PVPVA50 and PAA450 grades with a final $0.4 \mathrm{wt} \%$. 
As compared to Bond, deposits from the polymer mixtures retained a higher fraction of their AZ deposit after 1 washing with values more than 67 and 90\% for PVP and PVPVA mixtures, respectively, when the corresponding value for Bond was only 50\%. This demonstrates the slow diffusion rate of water into those strong polymer complexes. From the swelling-dissolution data, the diffusion coefficient, $D\left(\mathrm{~cm}^{2} \mathrm{sec}^{-1}\right)$ for the transport of water molecules in film sample can be determined empirically as $\pi\left(\frac{x \cdot r}{4 \cdot s}\right)^{2}$, where $\mathrm{x}$ is the initial thickness of film (Table S4), $r$ is the slope of the linear part of the swelling time course and $\mathrm{s}$ is the maximum swelling at time $\mathrm{t}$ after immersion of film in water [42]. The above equation assumes constant diffusivity (steady-state relaxation-swelling rate) where dissolution is negligible. Also, it should be noted that natural rainwater has a slightly acidic $\mathrm{pH}\left(\mathrm{pH}_{\text {rain }} \approx 5.6\right)$, which is below the $\mathrm{pH}$ of deionized water $(\approx 7)$ employed in our studies. This means that our experiments may underestimate the rainfastness of PVP-PAA blends under field conditions where the rainwater is more acidic and the polymer complexes are expected to dissolve slower as the $\mathrm{pH}_{\text {rain }}$ approaches $\mathrm{pH}_{\text {crit }}(\approx 4.1)$.

Drop-on-drop method. In situ polymeric complexation is a new approach recently introduced by Damak et al [23] for reducing problems related to agrochemical spray bounce-off. In contrast to the pre-formed complexes, the solution-to-insoluble complex transition occurs by physical reaction of the two oppositely charged polymers simultaneously sprayed on plant surfaces. Similarly, interpolymer complexes can be formed in situ by simple precipitation on plant surfaces as a novel method for enhancing agrochemical rainfastness. This is also similar to hydrogenbonded layer-by-layer deposition method employed in multilayered bioadhesive formulations [43, 44]. Here, we used this method by making drop-on-drop impacts with the two polymer solutions (PVP or PAA) so that a combined drop $(10 \mu \mathrm{L})$ with a 50:50 weight ratio can be formed on paraffin film. 
The results in Figure 8 show that when a PVP drop first impacts the surface followed by PAA a striking rainfastness increase ( $\mathrm{p}<0.05$, Dunnett's post hoc test) almost to the extent of PVOH-HM and Bond is observed. This results in at least 10-times enhanced effect than without PVP and 2times for the pre-mixtures.

A
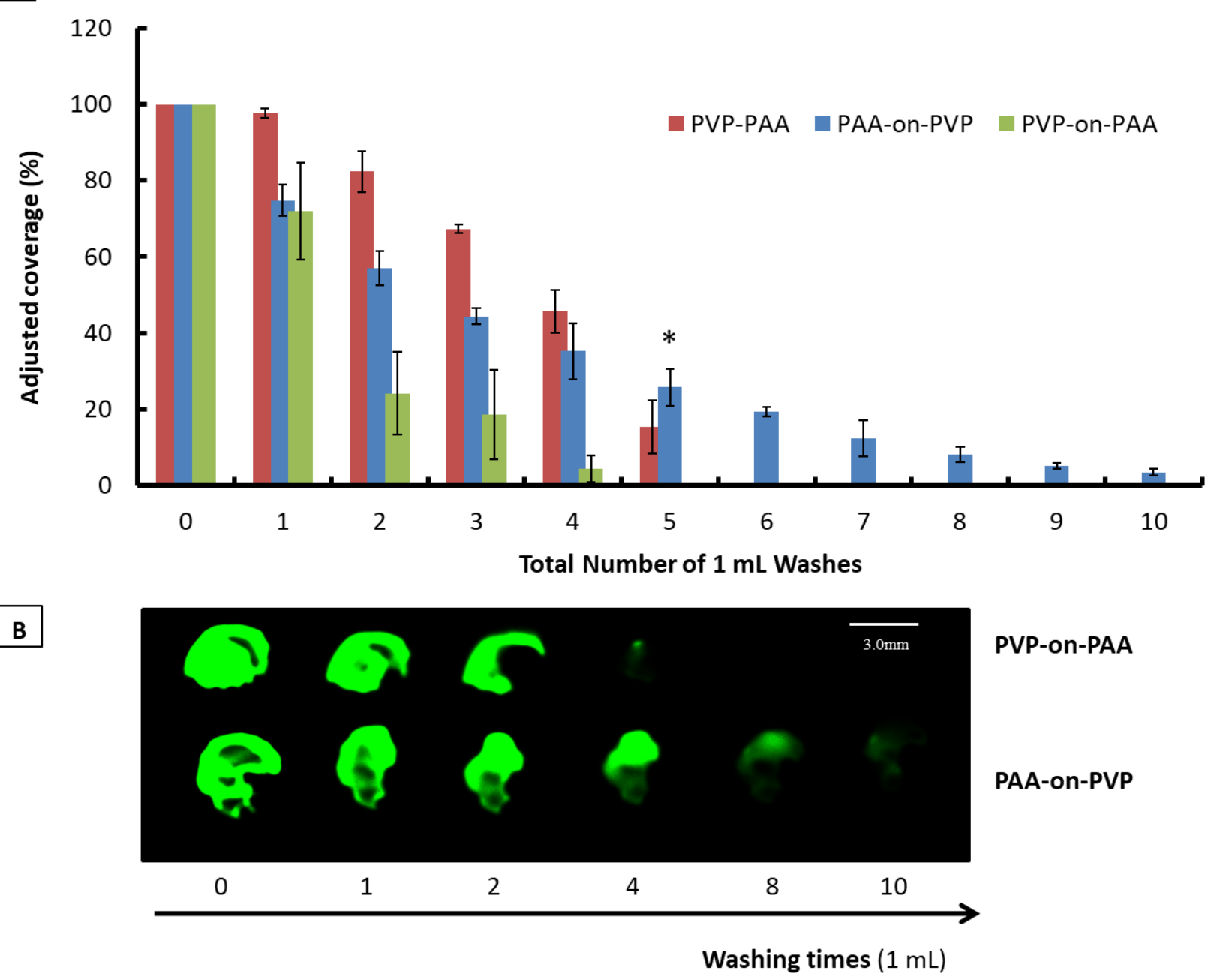

PVP-on-PAA

PAA-on-PVP

Fig. 8. A. Wash-off of the drop-on-drop impacts on paraffin film surfaces compared to the premixture PVP-PAA. Measurements are presented as the mean $(n=3)$ fluorescent spread area \pm standard deviation of the deposited AZ particles after processed with ImageJ. * No significant difference $(p>0.05$, Independent-samples T-test). B. Representative FM images of the drop-on- 
drop impacts showing their washing-off pattern during simulated rain washes. Mixture solutions refer to PVP1300 and PAA450 grades mixed at $\Phi w=50: 50$ with a final $0.4 \mathrm{wt} \%$.

It appears also that loss of $\mathrm{AZ}$ is significantly higher $(\mathrm{p}<0.05$, independent samples $\mathrm{T}$-test) after the first 3 washing times (around 22.7, 24.1 and 23\% more wash-off, respectively) than in mixtures. The explanation can be given is based on the differences in washing-off of the fraction of fungicide particles uncovered by interpolymer complexes network. This hypothesis supports the patterns observed in fluorescent microscopy images (Figure 8B) showing the irregular distribution of AZ particles in drop-on-drop deposits probably since the two drops left to physically interact on paraffin film with no mixing. When a PAA drop first hits the surface, however, washedoff erosion is significantly more pronounced $(\mathrm{p}<0.05$, independent samples $\mathrm{T}$-test) than the premixed polymers, indicating the significance of the order of polymer impaction on interpolymer complexes adhesion.

In both cases, it is assumed that the association of PVP with PAA will proceed at the same degree primarily attributed to hydrogen bonding interactions between PVP and PAA and secondary hydrophobic interactions [21]. Thus, it is expected that both impacts to show similar cohesive interactions, although expressed with a different dynamics as postulated by the higher diffusion coefficient of PVP than PAA (see Figure S1). That implies that at the equilibrium the difference in rainfastness activity can be attributed mainly to differences in the strength of the adhesive joint made between the drop and paraffin film. PVP is more hydrophobic [34] and might have stronger affinity than PAA for paraffin film (data not shown), implying that PAA-on-PVP drop impacts lead to stronger adhesion than their counterparts. This might account for the observation that PVPon-PAA deposits failed more adhesively than cohesively as washing-off events eroded the deposits 
by removing them as large undissolved masses (data not shown). This may have a practical importance as the splashing rain on fungicide deposit may increase fungicide efficacy by redepositing the active particles in unprotected areas [6].

Rainfastness of different PVP grades and mixtures were evaluated on Vicia faba leaves under the similar method described for paraffin film. The selection of the particular plant species was based on the smooth type surface of its leaves. The latter aimed to eliminate any differences in the effects due to the surface roughness as paraffin film surface is smooth. Generally, similar results were noticed (Figure S12) with the difference that the washing-off resistance on leaves was 1-2 orders of magnitude weaker. This is likely ascribed to the complex chemical and microroughness nature of plant leaves which can negatively affect the adhesion [45]. Regardless their chemical nature, both paraffin film and leaf exhibit similar hydrophobicity, however, leaves also exhibit measurable polar forces including hydrogen bonding or/and acid-base interactions (Table S2). This seems to give a reasonable explanation for the greater rainfastness of PVP drop-on-PAA drop impacts reported on leaves than paraffin film owing possibly to the specific interactions developed between the polyelectrolyte and the leaf surface.

\subsection{SEM analysis}

SEM examination (Figure 9) of the planar topography of the deposits revealed the presence of irregular fibrous structures in drop-on-drop impacts likely linked to the formation of interpolymer complexes. In contrast, deposits containing premixed polymers gave a more uniform morphology with no indication of immiscibility. Deposits by the PVP-on-PAA drop method showed extending delamination as indicated the existence of a loose adhesive interface between the deposit and the 
paraffin film surface, corroborating the idea that PAA may weaken drop adhesion, which in turn may render the displacement of deposit from paraffin film by water easier.
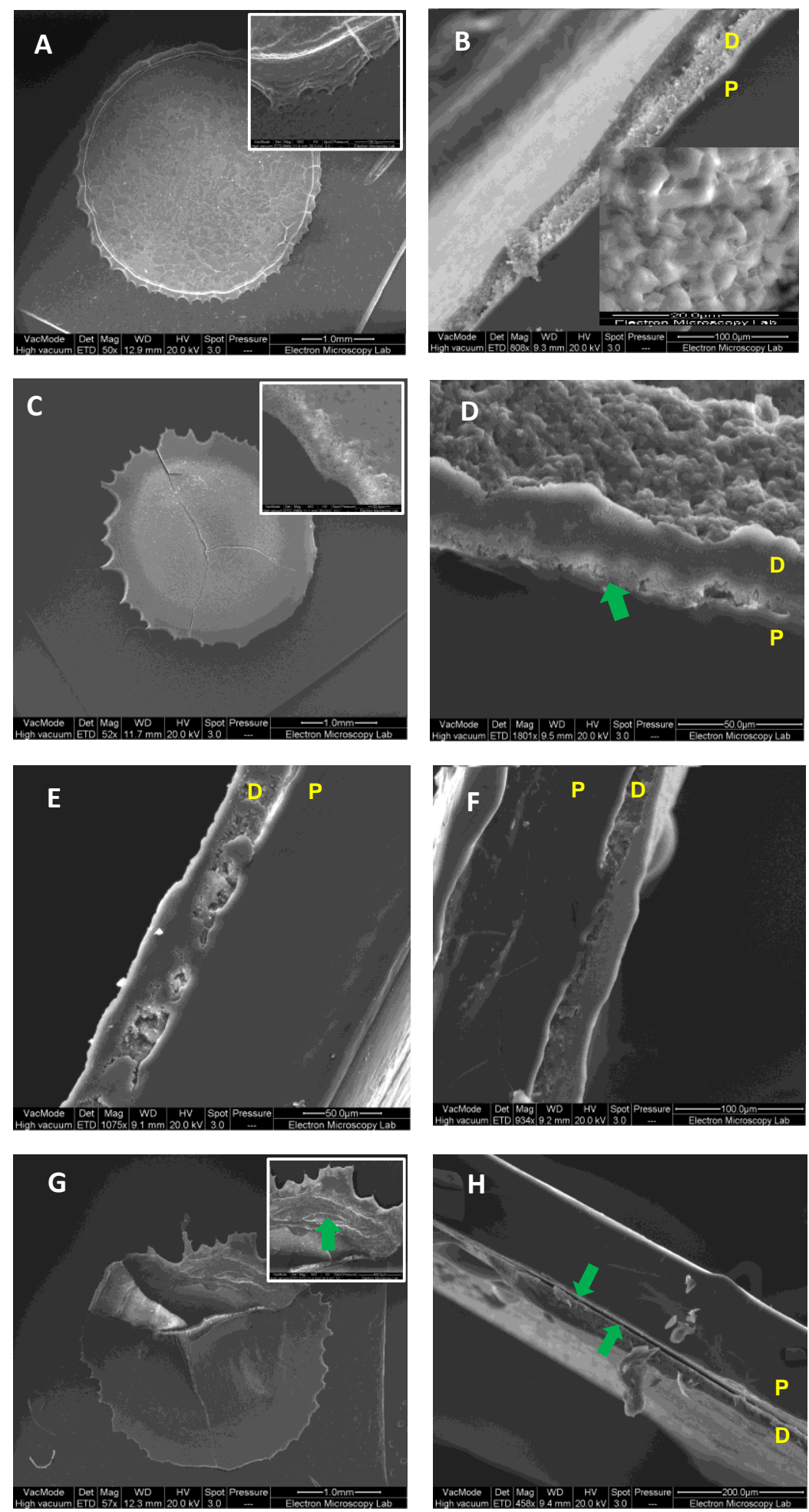
Fig. 9. Representative SEM images of planar and cross-section topography of PVP deposits (D) on paraffin film $(\mathrm{P})$. Deposits $(10 \mu \mathrm{L})$ were placed on paraffin film and left to evaporate for $1.5 \mathrm{hr}$ at $20^{\circ} \mathrm{C}$. A. Planar topography of AZ dry deposit indicating the high fraction of fungicide particles accumulated at the periphery of the deposit (insert). B. Cross-section of the AZ deposit indicating the loosely bound fungicide particles on the surface. Insert shows the fungicide particle size. C. Planar topography of PVP1300 deposit showing that fewer particles are accumulated at the periphery (insert). D. Adhesive interface at the cross section of PVP1300. Note the artefact of "hybrid layer" formed at the adhesive interface (green arrowhead) assigned possibly due to the sample preparation step. E. Adhesive interface at the cross-section of PVP-PAA mixture deposits. F. Adhesive interface of deposits after PAA-on-PVP drop impacts. G. Planar topography of deposits after PVP-on-PAA drop impacts. Note also the fibrous-like structures (green arrowhead in the inset), which were assigned to precipitates of interpolymer complexes. H. Cross-section of the deposit after PVP-on-PAA drop impacts showing the weak adhesive interface (green arrowheads) between the dry deposit and the paraffin film.

Also, despite some artefact "hybrid" interface noticed (which probably occurred during the cutting procedure of the samples) there was no indication of a real "hybrid" like layer formed at the interface which could indicate the involvement of binding between the bulk of the deposit and the paraffin film.

The scenario arising from the above studies can be schematically illustrated in Figure 10A. The molecular weight (Figure 9A) affects the tenacity of PVP deposits by affecting both the cohesive and adhesive phenomena. Regarding the adhesiveness, higher polymer molecular weight (up to a certain length) may increase bioadhesive forces. The above means that an increase in the molecular weight of macromolecules increases the interpenetration layer and molecular entanglements of the 
polymer with the substrate [46]. Regarding the cohesive forces, dissolution tests with PVP films confirm that the lower the Mw the faster their dissolution.

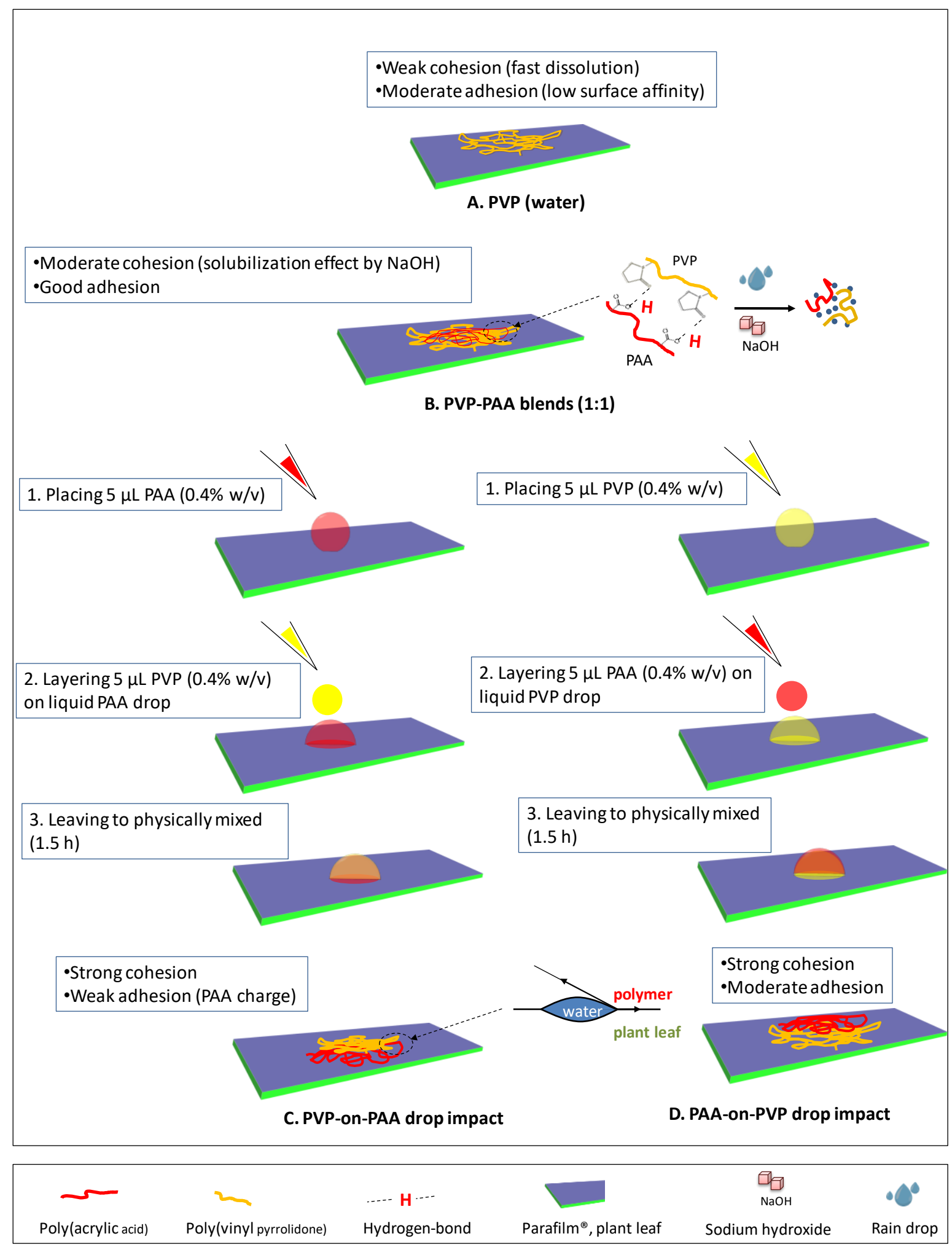


Fig. 10. Schematic illustration of PVP rainfastness on model hydrophobic surfaces using different formulation systems. A. Simple physical adhesion of PVP (physisorption) exerted by its molecular weight. B. Enhancing PVP rainfastness by mixing with PAA. C, D. In situ polymer interactions in drop impacts of PVP-on-PAA (C) and PAA-on-PVP (D) systems.

PVP-PAA blends (Figure 9B) exhibit enhanced tenacity as a result of the complexation between the two polymers. Formation of IPC affects the cohesive energy of the hydrophilic PVP leading to a decrease in the diffusion of water into the deposit. There is an optimum 50:50 stoichiometric weight ratio where films also showed enhanced thermodynamic properties. However, these formulations exhibit moderate rainfastness activity, which is likely attributed to the presence of $\mathrm{NaOH}$ accelerating deposit wash-off.

The order of addition of the two polymers onto the surface may have a dramatic impact on the tenacity. When PVP first settles on the surface (Figure 9C), deposits show an enhanced rainfastness like the controls. In the reverse order (Figure 9D), deposits washed-off more easily than the premixtures, indicating extensive adhesive failure.

\section{Conclusions}

Three approaches were investigated to enhance the rainfastness properties of the water-soluble polymer PVP using fluorescent microscopy analysis of dried deposits containing the fluorescent compound azoxystrobin. First, it was found that the highest molecular weight grades of the polymer correlated to a higher rainfastness and slow film dissolution profile in water. Despite that, PVP was not as good as other insoluble polymers such as PVOH and the commercial sticker adjuvant Bond. It was shown that the faster dissolution rate and the weak adhesion of PVP on 
hydrophobic model surfaces as compared to the controls give an explanation about that failure. In a second approach, the rainfastness of PVP was significantly improved by appropriate mixing the polymer with PAA which has ability to form insoluble complexes with PVP. Formation of interpolymer complexes affects the cohesive energy of the hydrophilic PVP leading to a decrease in the diffusion coefficient of water into the deposit and thus rendering azoxystrobin more rainfast. In a third approach, a novel method was used by making drop impacts of PVP-on-PAA or PAAon-PVP. This method allows the in situ formation of IPC between the two polymers and tackles the issue of solubilization effect from the presence of $\mathrm{NaOH}$ in pre-mixtures. Interestingly, it was found that the order of addition of the two polymers was of paramount importance. Greater rainfastness activity was revealed by adding first PVP and second PAA drops, while in the case of the reverse order deposits showed excessive washing-off. Potentially in situ formation of deposits on leaf surfaces could be achieved by using sequential spraying of agrochemicals.

This work gives an insight into the mechanisms underlying rainfastness which may help to guide the utilization of novel approaches to manipulate rainfastness of water-soluble polymers as sticker adjuvants in crop protection. The proof-of-concept study of using PVP-PAA mixtures may open a new "smart" delivery method in agrochemical formulations and depending on the field conditions tailored sticker adjuvants may be designed to respond to environmental stimuli such as $\mathrm{pH}$, ionic strength and temperature.

\section{Supporting Information}

$\mathrm{pH}$ critical values, surface tension and physicochemical characteristics for aqueous PVP solutions and their cast films. DLS analysis of PVP and PAA aqueous solutions. Estimation of 
the affinity of polymers for paraffin film surfaces. The following files are available free of charge.

\section{Author Contributions}

The manuscript was written through contributions of all authors. All authors have given approval to the final version of the manuscript.

\section{Notes}

The authors declare no competing financial interest.

\section{Acknowledgment}

We are grateful to Syngenta Ltd for providing the fungicide azoxystrobin, growing and supplying Vicia faba plants used. We also thank the Chemical Analysis Facility (CAF) at the University of Reading for the use of DSC, TGA and SEM instruments.

\footnotetext{
Abbreviations

PVP; poly(vinyl pyrrolidone), PVPVA; poly(vinyl pyrrolidone-co-vinyl acetate), PAA; poly(acrylic acid), IPC; Interpolymer Complexation, AZ; azoxystrobin; Tg; glass transition temperature, $\Delta \mathrm{H}$, enthalpy change, FM; Fluorescent Microscopy, SEM; Scanning Electron Microscopy, DSC, Differential Scanning Calorimetry, DLS; Diffraction Light Scattering.
} 


\section{References}

[1] M. Hunsche, L. Damerow, M. Schmitz-Eiberger and G. Noga, Mancozeb wash-off from apple seedlings by simulated rainfall as affected by drying time of fungicide deposit and rain characteristics, Crop Prot. 26 (2007) 768-774.

[2] J. Zabkiewicz, Adjuvants and herbicidal efficacy-present status and future prospects, Weed Res. 40 (2000)139-149.

[3] R. Gaskin, K. Steele, A comparison of sticker adjuvants for their effects on retention and rainfastening of fungicide sprays, N. Z. Plant Prot., 62 (2009) 339-342.

[4] C. Yao, K. Myung, N. Wang and A. Johnson, Retention, Uptake, and Translocation of Agrochemicals in Plants, ACS Publications (2014) pp 1-22.

[5] M. Khayet, M. and V. Fernández, Estimation of the solubility parameters of model plant surfaces and agrochemicals: a valuable tool for understanding plant surface interactions, Theor. Biol. Med. Model., 9 (2012) 1- 21.

[6] E. Hislop and T. Cox, Local redistribution of fungicides on leaves by water, Ann. Appl. Biol., 66 (1970) 89-101.

[7] B. Shasha, and M. McGuire, Sprayable gluten-based formulation for pest control, U.S. Patent 5,505,940. (1996). 
[8] M.T. Meredith, A.J. Stern and D. Saylik, Polyamide and polyimide sticker adjuvants U.S. Patent 9,668,472. U.S. Patent 9,668,472 (2017).

[9] E.C. Kostansek, Dithiocarbamate compositions having increased rain fastnes.U. Patent 0,245,970. (1987).

[10] A.R. Clark and M.M Clark, Compositions and method for controlling release of pesticides using terpene polymers, U.S. Patent 3,592,910. (1971).

[11] S.D. Desai, S.E. Bhoge, J.R. Shroff and V.R. Shroff, Agrochemical compositions having increased rainfastness, U.S. Patent 20160270392. (2014).

[12] P.K. Leonard, Method for the enhancement of the residual activity of pesticide formulations, E.U. Patent 0,981,957. (2000).

[13] A.K. Underwood, Adjuvant Trends for the New Millennium 1, Weed Technol., 14 (2000) $765-772$.

[14] B.L. Symonds, C.I. Lindsay, N.R. Thomson and V.V. Khutoryanskiy, Chitosan as a rainfastness adjuvant for agrochemicals, RSC Adv., 6 (2016) 102206-102213. 
[15] B.L. Symonds, N.R. Thomson, CI. Lindsay and V.V. Khutoryanskiy, Rainfastness of Poly(vinyl alcohol) Deposits on Vicia faba Leaf Surfaces: From Laboratory-Scale Washing to Simulated Rain, ACS Appl. Mater. Interfaces., 8 (2016) 14220-14230.

[16] P. Steward, J. Hearn and M. Wilkinson, An overview of polymer latex film formation and properties, Adv. Colloid. Interface. Sci., 86 (2000) 195-267.

[17] V. Bühler (Ed.), Polyvinylpyrrolidone excipients for pharmaceuticals: povidone, crospovidone and copovidone, Springer Science \& Business Media (2005).

[18] K.S. Narayanan, S.L. Paul and R.K. Chaudhuri, N-alkyl pyrrolidones for superior agricultural adjuvants, Pest. Manag. Sci., 37 (1993) 225-228.

[19] L. C. Cesteros, E. Meaurio and I. Katime, Formation of interpolymer complexes between poly(monoethyl itaconate) and poly(N -vinyl-2-pyrrolidone), Polym. Int., 34 (1994), 97-103.

[20] C. Maltesh, P. Somasundaran, R. Kulkarni and S. Gundiah, Polymer-polymer complexation in dilute aqueous solutions: Poly(acrylic acid)-poly(ethylene oxide) and poly(acrylic acid)-poly(vinylpyrrolidone), Langmuir, 7 (1991) 2108-2111.

[21] Z.S. Nurkeeva, G.A. Mun, V.V. Khutoryanskiy, A.B. Bitekenova, A.V. Dubolazov, and S.Z. Esirkegenova, $\mathrm{pH}$ effects in the formation of interpolymer complexes between poly (N- 
vinylpyrrolidone) and poly (acrylic acid) in aqueous solutions, Eur. Phys. J. E Soft. Matter., $10(2003) 65-68$.

[22] D.E. Zhunuspayev, G.A. Mun, P. Hole and V.V. Khutoryanskiy, Solvent effects on the formation of nanoparticles and multilayered coatings based on hydrogen-bonded interpolymer complexes of poly(acrylic acid) with homo- and copolymers of $\mathrm{N}$-vinyl pyrrolidone, Langmuir, 24 (2008) 13742-13747.

[23] M. Damak, S.R. Mahmoudi, M.N. Hyder, and K.K. Varanasi, Enhancing droplet deposition through in-situ precipitation, Nat.Commun., 7 (2016) 1-9.

[24] C. Huck-Iriart, A. De-Candia, J. Rodriguez and C. Rinaldi, Determination of Surface Tension of Surfactant Solutions through Capillary Rise Measurements: An Image-Processing Undergraduate Laboratory Experiment, J. Chem. Educ., 9 (2016) 1647-1651.

[25] CIPAC, CIPAC Handbook-Volume L, CIPAC Ltd: Harpenden, UK (2005).

[26] I. Iliopoulos, J. Halary and R. Audebert, Polymer complexes stabilized through hydrogen bonds. Influence of "structure defects" on complex formation: Viscometry and fluorescence polarization measurements, J.Polym. Sci., Part A, 26 (1988) 275-284.

[27] S. Bucak and D. Rende, Colloid and Surface Chemistry: A Laboratory Guide for Exploration of the Nano World, CRC Press, 2013, p. 125- 186. 
[28] P.D. Lancashire, H. Bleiholder, T. Boom, P. Langelüddeke, R. Stauss, R., E.Weber and A. Witzenberger, A uniform decimal code for growth stages of crops and weeds, Ann. Appl. Biol., 119 (1991) 561-601.

[29] A. I. Isayev (Ed), Encyclopedia of polymer blends, Wiley-VCH Verlag \& Co. (2011), pp. 27-108.

[30] B.A. Miller-Chou and J.L. Koenig, A review of polymer dissolution, Prog.Polym. Sci., 28 (2003) 1223-1270.

[31] D. Lubasova, H. Niu, X. Zhao, and T. Lin, Hydrogel properties of electrospunpolyvinylpyrrolidone and polyvinylpyrrolidone/poly (acrylic acid) blend nanofibers, RSC Adv., 5 (2015) 54481-54487

[32] V.V. Khutoryanskiy, G.A. Mun, Z.S. Nurkeeva and A.V. Dubolazov, pH and salt effects on interpolymer complexation via hydrogen bonding in aqueous solutions, Polym. Int., 53 (2004) 1382-1387.

[33] H. Kaczmarek, A. Szalla and A. Kamińska, Study of poly (acrylic acid)-poly (vinylpyrrolidone) complexes and their photostability, Polymer, 42 (2001) 6057-6069. 
[34] A. Leiva, L. Gargallo, D. Radić, Interpolymer complexes. Properties and characterization, Polym.Int., 34 (1994) 393-396.

[35] A. Pizzi and K. L. Mittal (Eds.), Handbook of Adhesive Technology, Revised and Expanded, CRC Press (2003), pp. 957- 970.

[36] A I. Isayev (Ed), Encyclopedia of Polymer Blends, Vol. 3, VCH-Verlag (2016), pp. 1- 122.

[37] G.P. Kumar, A. Phani, R. Prasad, J.S. Sanganal, N. Manali, R.Gupta, N. Rashmi, G. Prabhakara and C.P. Salins, Sandeep, K., Polyvinylpyrrolidone oral films of enrofloxacin: Film characterization and drug release, Int. J. Pharmaceut., 471 (2014) 146-152.

[38] V. V. Khutoryanskiy, M. G. Cascone, L. Lazzeri, N. Barbani Z. S. Nurkeeva, G. A. Mun A. B. Bitekenova and A. B. Dzhusupbekova, Hydrophilic Films Based on Blends of Poly(acrylic acid) and Poly(2-hydroxyethyl vinyl ether): Thermal, Mechanical, and Morphological Characterization, Macromol. Biosci., 3 (2003), p. 117- 122.

[39] I. Rainaldi, C. Cristallini, G. Ciardelli and P. Giusti, Kinetics and reaction mechanism of template polymerization investigated by conductimetric measurements. Part 3. Radical polymerization of acrylic acid in the presence of poly( $\mathrm{N}$-vinylpyrrolidone), Polym. Int. 49 (2000), p. 63-73. 
[40] P. Irja (Ed), Polymeric surfactants, Surfactant science series, Vol 42, CRC Press (1992), pp. 107- 125.

[41] A.F.M. Barton (Ed), Handbook of solubility parameters and other cohesion parameters, CRC Press (1991), pp. 583- 630.

[42] J. Mudassir and N. M. Ranjha, Dynamic and equilibrium swelling studies: cross-linked pH sensitive methyl methacrylate-co-itaconic acid (MMA-co-IA) hydrogels, J. Polym. Res., 15 (2007) 195-203.

[43] E. Kharlampieva and S.A. Sukhishvili, Hydrogen-bonded layer-by-layer polymer films, J.Macromol. Sci, Part C, 46 (2006) 377-395.

[44] E. Kharlampieva, V. Kozlovskaya, S.A. Sukhishvili, Layer-by-Layer Hydrogen-Bonded Polymer Films: From Fundamentals to Applications, Adv. Mater., 21 (2009) 3053-3065.

[45] J.J. Nairn, W.A. Forster, and R.M. van Leeuwen, Quantification of physical (roughness) and chemical (dielectric constant) leaf surface properties relevant to wettability and adhesion, Pest. Manag. Sci., 67 (2011) 1562-1570.

[46] R Gurny, J M Meyer and N A Peppas, Bioadhesive Intraoral Release Systems: Design, Testing and Analysis, Biomaterials, 5 (1984) 336-340. 
Graphical abstract

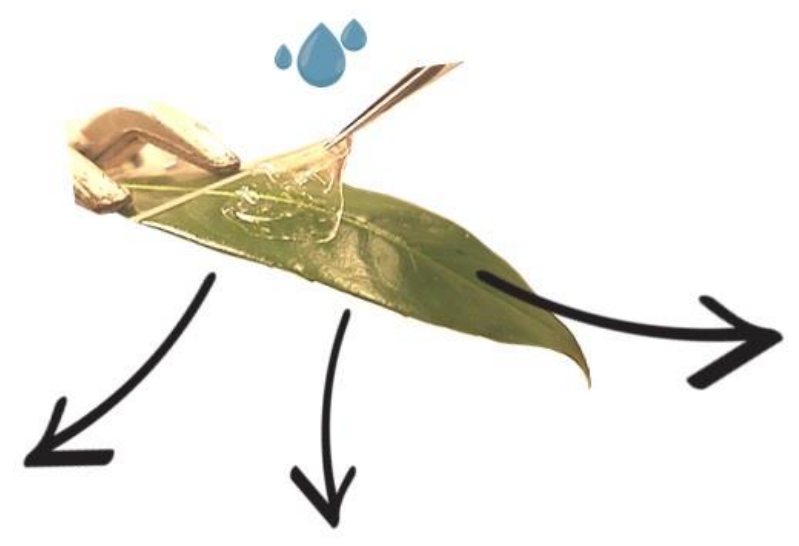

C. PAA-on-PVP drop impacts

A. PVP

B. PVP-PAA blends
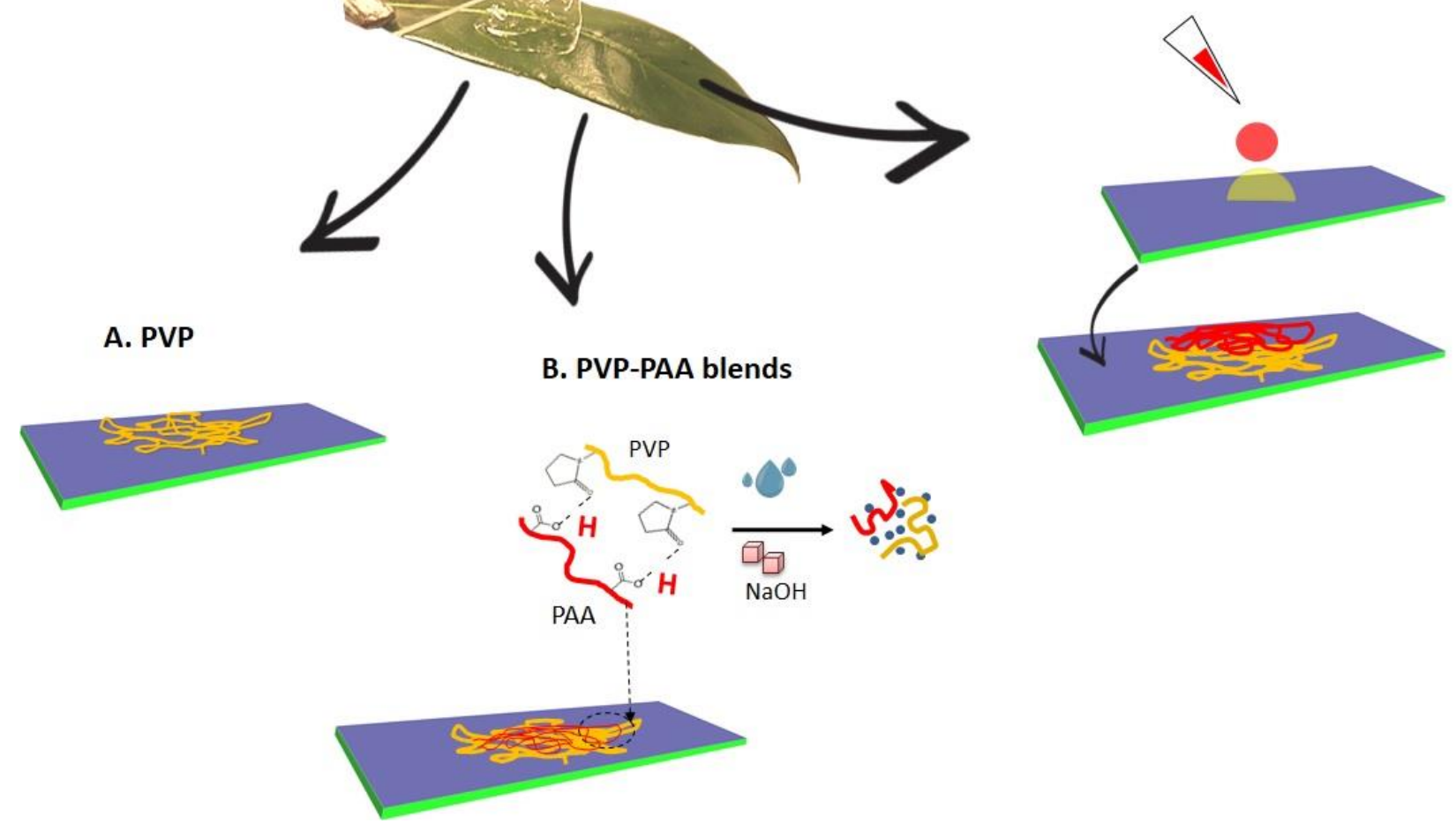